\title{
Fuzzy Assessment Methods
}

\author{
Igor Ya. Subbotin ${ }^{1}$, Michael Gr. Voskoglou ${ }^{2}$ \\ ${ }^{1}$ Department of Mathematics and Natural Sciences, College of Letters and Sciences, National University, USA \\ ${ }^{2}$ Department of Mathematical Sciences, School of Technological Applications, Graduate Technological Educational Institute (T. E. I.) of \\ Western Greece, Greece
}

Copyright (C) 2014 Horizon Research Publishing All rights reserved.

\begin{abstract}
Fuzzy logic due to its nature of characterizing each case with multiple values offers is a rich field of resources covering the assessment of situations characterized by a degree of fuzziness and/or uncertainty. In this paper, we present two different fuzzy assessment models: The Centre of Gravity (COG) model and Trapezoidal Fuzzy Assessment Model (TRFAM). The last one is a new model covering the ambiguous cases being at the boundaries between two successive assessment intervals. An application (students' assessment) is also presented, through which the above methods are compared to each other and also with two traditional assessment methods based on principles of bivalent logic (the calculation of means and of the GPA index). Some useful conclusions are drawn about the characteristics of the above fuzzy assessment models.
\end{abstract}

Keywords GPA Index, Fuzzy Sets, Centre of Gravity (COG) Defuzzification Technique, Trapezoidal Fuzzy Assessment Model (TRFAM)

\section{Introduction}

The assessment of a system's effectiveness (i.e. of the degree of attainment of its targets) with respect to an action performed within the system (e.g. problem-solving, decision making, learning performance, etc) is a very important task that enables the correction of the system's weaknesses resulting to the improvement of its general performance.

The assessment methods that are commonly used in practice are based on the principles of the classical, bivalent logic (yes-no). However, there are cases where a crisp characterization is not probably the proper one for the assessment. For example, a teacher is frequently not sure about a particular numerical grade characterizing a student's performance. Fuzzy logic, due to its nature of characterizing a case with multiple values, offers wider and richer resources covering such kind of cases.

The several methods in use for assessing a system's performance focus on different targets: Some of them measure the mean system's performance, while others focus to its quality performance by assigning greater coefficients (weights) to the higher scores. Therefore, one who wants to obtain a comprehensive view of a system's performance must make use of more than one assessment methods for this purpose.

In this paper we propose two different fuzzy assessment models focusing to the quality performance of a system, including the newly created trapezoidal model. These methods are developed in the next two sections of the paper, namely the centre of gravity (COG) or centroid defuzzification model (Section 2), and the trapezoidal fuzzy assessment model (TRFAM) (Section 3). Some applications presented in Section 4 illustrating our results in practice. The above two methods are compared there to each other and to other traditional methods (calculation of the means and of the GPA index). We are concluding by summarizing in Section 5 the main results and describing our future plans for further research on the subject. For general facts on fuzzy sets we refer to the book [2].

\section{Use of the COG Defuzzification Technique as an Assessment Method}

The COG technique is a very popular in fuzzy mathematics defuzzification method (e.g. see [10]). For applying this technique we correspond to each $x$ of the universal set $U$ an interval of values from a prefixed numerical distribution, which actually means that we replace $\mathrm{U}$ with a set of real intervals. Then, we construct the graph of the corresponding membership function $y=m(x)$. There is a commonly used in fuzzy logic approach to represent the fuzzy data with the pair of numbers $\left(x_{c}, y_{c}\right)$ as the coordinates of the COG, say $\mathrm{F}_{\mathrm{c}}$, of the level's section $S$ contained between the above graph and the OX axis, which we can calculate using the following well-known (e.g. see [15]) formulas:

$$
x_{c}=\frac{\iint_{S} x d x d y}{\iint_{S} d x d y}, y_{c}=\frac{\iint_{S} y d x d y}{\iint_{S} d x d y}
$$


In earlier papers Subbotin and Voskoglou have properly adapted the COG technique to be used as an assessment method, e.g. see $[3,9,10,11,12]$, etc. In fact, let $\mathrm{G}$ be a group of individuals participating in a certain activity and let $\mathrm{U}=\{\mathrm{A}, \mathrm{B}, \mathrm{C}, \mathrm{D}, \mathrm{F}\}$ be a set of linguistic labels (grades) characterizing the individuals' performance with respect to this activity as follows: $\mathrm{A}=$ excellent, $\mathrm{B}=$ very good, $\mathrm{C}=$ good, $\mathrm{D}=$ moderate (satisfactory) and $\mathrm{F}=$ unsatisfactory. Then, we can express $\mathrm{G}$ as a fuzzy set in $\mathrm{U}$ in the form $\mathrm{G}=\{(x, m(x)), x$ $\in \mathrm{U}\}$, where $y=m(\mathrm{x})$ is the corresponding membership function.

We correspond to each $x$ in $\mathrm{U}$ an interval of real values as follows: $\mathrm{F} \rightarrow[0,1), \mathrm{D} \rightarrow[1,2), \mathrm{C} \rightarrow[2,3), \mathrm{B} \rightarrow[3,4)$, $\mathrm{A} \rightarrow[4,5]$. Consequently, we have that $y_{1}=m(x)=m(\mathrm{~F})$ for all $x$ in $[0,1), y_{2}=m(x)=\mathrm{m}(\mathrm{D})$ for all $x$ in $[1,2), y_{3}=m(x)$ $=m(\mathrm{C})$ for all $x$ in $[2,3), y_{4}=m(\mathrm{x})=m(\mathrm{~B})$ for all $x$ in $[3,4)$ and $y_{5}=m(x)=m(\mathrm{~A})$ for all $x$ in $[4,5]$. Then the graph of the membership function $y=m(x)$, takes the form of the bar graph of Figure 1, while the area of the level's section $S$ contained between this graph and the $\mathrm{OX}$ axis is equal to the sum of the areas of the rectangles $\mathrm{S}_{\mathrm{i}}, \mathrm{i}=1,2,3,4,5$.

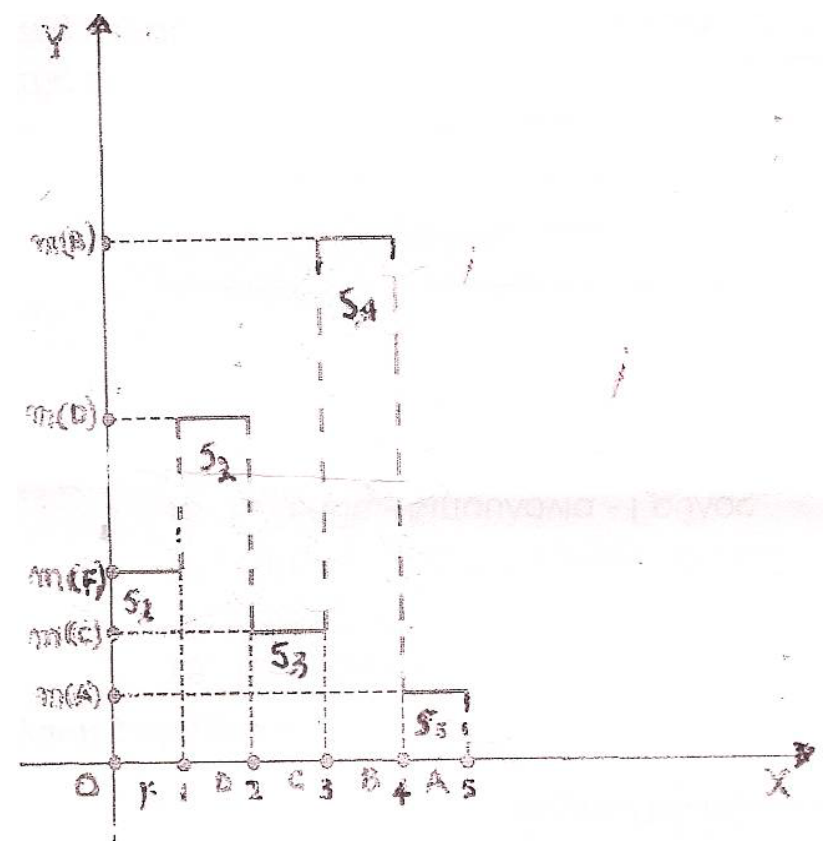

Figure 1. Bar graphical data representation

It is straightforward then to check that in this case formulas (1) are transformed to the form:

$$
\begin{aligned}
& x_{c}=\frac{1}{2}\left(\frac{y_{1}+3 y_{2}+5 y_{3}+7 y_{4}+9 y_{5}}{y_{1}+y_{2}+y_{3}+y_{4}+y_{5}}\right), \\
& y_{c}=\frac{1}{2}\left(\frac{y_{1}^{2}+y_{2}^{2}+y_{3}^{2}+y_{4}^{2}+y_{5}^{2}}{y_{1}+y_{2}+y_{3}+y_{4}+y_{5}}\right)
\end{aligned}
$$

with $y_{i}=m\left(x_{i}\right), \mathrm{i}=1,2,3,4,5$ and $x_{1}=\mathrm{F}, x_{2}=\mathrm{D}, x_{3}=\mathrm{C}, x_{4}=\mathrm{B}$ and $\mathrm{x}_{5}=\mathrm{A}$.
In fact, $\iint_{S} d x d y$ is the area of $\mathrm{S}$ which is equal to $\sum_{i=1}^{5} y_{i}$,

$$
\begin{gathered}
\text { Also } \iint_{S} x d x d y=\sum_{i=1}^{5} \iint_{S} x d x y=\sum_{i=1}^{5} \int_{0}^{y} d y \int_{i=1}^{i} x d x \\
=\sum_{i=1}^{5} y_{i} \int_{i=1}^{i} x d x=\sum_{i=1}^{5} y_{i}\left[\frac{x^{2}}{2}\right]_{i-1}^{i}=\frac{1}{2} \sum_{i=1}^{5} y_{i}\left[i^{2}-(i-1)^{2}\right]= \\
\frac{1}{2} \sum_{i=1}^{5}(2 i-1) y_{i}
\end{gathered}
$$

and $\iint_{S} y d x d y=$

$$
\begin{gathered}
\sum_{i=1}^{5} \iint_{F_{i}} y d x d y=\sum_{i=1}^{5} \int_{0}^{y_{i}} y d y \int_{i-1}^{i} d x= \\
\sum_{i=1}^{n} \int_{0}^{y_{i}} y d y=\frac{1}{2} \sum_{i=1}^{n} y_{i}^{2}
\end{gathered}
$$

Normalizing the membership degrees by dividing each $y_{i}$ by the $\operatorname{sum} \sum_{i=1}^{5} y_{i}$ we may assume without loss of generality that $\sum_{i=1}^{5} y_{i=1}$. Therefore formulas (2) can be finally written in the form:

$$
\begin{gathered}
x_{c}=\frac{1}{2}\left(y_{1}+3 y_{2}+5 y_{3}+7 y_{4}+9 y_{5}\right), \\
y_{c}=\frac{1}{2}\left(y_{1}{ }^{2}+\mathrm{y}_{2}{ }^{2}+y_{3}{ }^{2}+y_{4}{ }^{2}+y_{5}{ }^{2}\right)
\end{gathered}
$$

Next, using elementary algebraic inequalities it is easy to check that there is a unique minimum for $y_{c}$ corresponding to the COG $\mathrm{F}_{\mathrm{m}}\left(\frac{5}{2}, \frac{1}{10}\right)$ (an analogous process for TRFAM is developed in detail in paragraph 5 of section 3 ). Further, the ideal case is when $y_{1}=y_{2}=y_{3}=y_{4}=0$ and $y_{5}=1$. Then from formulas (3) we get that $x_{c}=\frac{9}{2}$ and $y_{c}=\frac{1}{2}$. Therefore the COG in this case is the point $F_{i}\left(\frac{9}{2}, \frac{1}{2}\right)$. On the other hand the worst case is when $y_{1}=1$ and $y_{2}=y_{3}=y_{4}=y_{5}=0$. Then from formulas (3) we find that the COG is the point $F_{w}\left(\frac{1}{2}, \frac{1}{2}\right)$.

Therefore the COG $F_{c}$ of the level's section $\mathrm{S}$ lies in the area of the triangle $\mathrm{F}_{\mathrm{w}} \mathrm{F}_{\mathrm{m}} \mathrm{F}_{\mathrm{i}}$.

Then by elementary geometric observations (an analogous process for TRFAM is developed in detail in paragraphs 5 and 6 of section 3) one can obtain the following criterion:

- Between two groups the group with the bigger $\mathrm{x}_{\mathrm{c}}$ performs better. 
- If the two groups have the same $\mathrm{x}_{\mathrm{c}} \geq 2.5$, then the group with the bigger $y_{c}$ performs better.

- If the two groups have the same $x_{c}<2.5$, then the group with the lower $y_{c}$ performs better.

As it becomes evident by the above presentation, the application of the COG method is simple in practice needing no complicated calculations in its final step.

\section{The Trapezoidal Fuzzy Assessment Model (TRFAM)}

The TRFAM is a recently developed $[6,7]$ new original variation of the COG method presented in the previous section. The important novelty of this approach is in the replacement of the rectangles appearing in the graph of the membership function of the COG method (Figure 1) by isosceles trapezoids sharing common parts, so that to cover the ambiguous cases of individuals' scores being at the limits between two successive grades. In the TRFAM's scheme (Figure 2) we have five trapezoids, corresponding to the individuals' performance characterizations $\mathrm{F}, \mathrm{D}, \mathrm{C}, \mathrm{B}$ and $\mathrm{A}$ respectively defined in the previous section. Without loss of generality and for making our calculations easier we consider isosceles trapezoids with bases of length 10 units lying on the OX axis. The height of each trapezoid is equal to the percentage of individuals who achieved the corresponding characterization for their performance, while the parallel to its base side is equal to 4 units. We allow for any two adjacent trapezoids to have $30 \%$ of their bases (3 units) belonging to both of them. In this way we cover the ambiguous cases of individuals' scores being at the limits between two successive grades. For students' assessment for example it is a very common approach to divide the interval of the specific grades in three parts and to assign the corresponding grade using + and - . For example, $75-77=$ B-, $78-81=\mathrm{B}, 82-84=\mathrm{B}+$. However, this consideration does not reflect the common situation, where the teacher is not sure about the grading of the students whose performance could be assessed as marginal between and close to two adjacent grades; for example, something like 84 - 85 being between $B_{+}$and $A_{-}$. The TRFAM fits this situation.

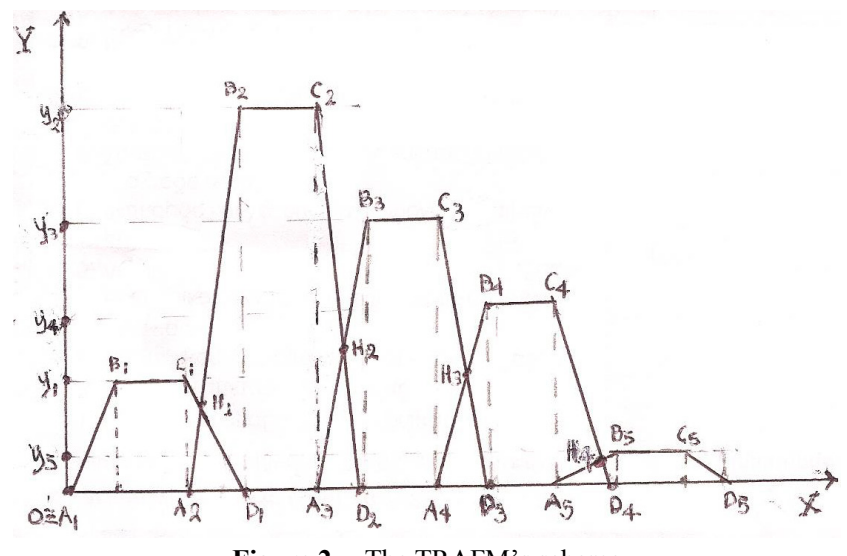

Figure 2. The TRAFM's scheme
An individuals" group can be represented, as in the COG method, as a fuzzy set in U, whose membership function $y=m(x)$ has as graph the line $\mathrm{OB}_{1} \mathrm{C}_{1} \mathrm{H}_{1} \mathrm{~B}_{2} \mathrm{C}_{2} \mathrm{H}_{2} \mathrm{~B}_{3} \mathrm{C}_{3} \mathrm{H}_{3} \mathrm{~B}_{4} \mathrm{C}_{4} \mathrm{H}_{4} \mathrm{~B}_{5} \mathrm{C}_{5} \mathrm{D}_{5}$ of Figure 2, which is the union of the line segments $\mathrm{OB}_{1}, \mathrm{~B}_{1} \mathrm{C}_{1}, \mathrm{C}_{1} \mathrm{H}_{1}, . ., \mathrm{B}_{5} \mathrm{C}_{5}$, $\mathrm{C}_{5} \mathrm{D}_{5}$. However, in case of the TRFAM the analytic form of $y$ $=m(x)$ is not needed for calculating the COG of the resulting area. In fact, since the marginal cases of the individuals' scores are considered as common parts for any pair of the adjacent trapezoids, it is logical to count these parts twice; e.g. placing the ambiguous cases $\mathrm{B}+$ and $\mathrm{A}$ - in both regions $\mathrm{B}$ and $\mathrm{A}$. In other words, the COG method, which calculates the coordinates of the COG of the area between the graph of the membership function and the $\mathrm{OX}$ axis, thus considering the areas of the "common" triangles $\mathrm{A}_{2} \mathrm{H}_{1} \mathrm{D}_{1}, \mathrm{~A}_{3} \mathrm{H}_{2} \mathrm{D}_{2}$, $\mathrm{A}_{4} \mathrm{H}_{3} \mathrm{D}_{3}$ and $\mathrm{A}_{5} \mathrm{H}_{4} \mathrm{D}_{4}$ only once, is not the proper one to be applied in the above situation.

Instead, in this case we represent each one of the five trapezoids of Figure 2 by its COG $F_{i}, i=1,2,3,4,5$ and we consider the entire area, i.e. the sum of the areas of the five trapezoids, as the system of these points-centers. More explicitly, the steps of the whole construction of the TRFAM are the following:

1. Let $y_{i}, \mathrm{i}=1,2,3,4,5$ be the percentages of the individuals' whose performance was characterized by $\mathrm{F}, \mathrm{D}, \mathrm{C}, \mathrm{B}$, and $\mathrm{A}$ respectively; then $\sum_{i=1}^{5} y_{i}=1(100 \%)$.

2. We consider the isosceles trapezoids with heights equal to $y_{i}, \mathrm{i}=1,2,3,4,5$, in the way that has been illustrated in Figure 2.

3. We calculate the coordinates $\left(x_{c_{i}}, y_{c_{i}}\right.$ ) of the COG $F_{i}, i=1,2,3,4,5$, of each trapezoid as follows: It is well known that the COG of a trapezoid lies along the line segment joining the midpoints of its parallel sides $a$ and $b$ at a distance $d$ from the longer side $b$ given by $d=\frac{h(2 a+b)}{3(a+b)}$, where $h$ is its height (e.g. see [14]). Therefore, in our case we have

$$
y_{c_{i}}=\frac{y_{i}(2 \cdot 4+10)}{3(4+10)}=\frac{3 y_{i}}{7}
$$

Also, since the abscissa of the COG of each trapezoid is equal to the abscissa of the midpoint of its base, it is easy to observe that $x_{c i}=7 i-2$.

4. We consider the system of the COG's $F_{i}, i=1,2,3,4,5$ and we calculate the coordinates $\left(\mathrm{X}_{\mathrm{c}}, \mathrm{Y}_{\mathrm{c}}\right)$ of the $\mathrm{COG} \mathrm{F}_{\mathrm{c}}$ of the whole area $S$ considered in Figure 2 by the following formulas, derived from the commonly used in such cases definition (e.g. see [15]): 


$$
X_{c}=\frac{1}{S} \sum_{i=1}^{5} S_{i} x_{c_{i}}, Y_{c}=\frac{1}{S} \sum_{i=1}^{5} S_{i} y_{c_{i}}
$$

In formulas (4) $S i, \mathrm{i}=1,2,3,4,5$ denote the areas of the corresponding trapezoids. Thus, $S i=\frac{(4+10) y_{i}}{2}=7 y_{i}$ and $S=\sum_{i=1}^{5} S_{i=7} \sum_{i=1}^{5} y_{i}=7$. Therefore, from formulas we finally get that

$$
\begin{aligned}
& X_{c}=\frac{1}{7} \sum_{i=1}^{5} 7 y_{i}(7 i-2)=\left(7 \sum_{i=1}^{5} i y_{i}\right)-2, \\
& Y_{c}=\frac{1}{7} \sum_{i=1}^{5} 7 y_{i}\left(\frac{3}{7} y_{i}\right)=\frac{3}{7} \sum_{i=1}^{5} y_{i}^{2}
\end{aligned}
$$

5. We determine the area where the COG $\mathrm{F}_{\mathrm{c}}$ lies as follows: For $\mathrm{i}, \mathrm{j}=1,2,3,4,5$, we have that $0 \leq\left(y_{i}-y_{j}\right)^{2}=y_{i}^{2}+y_{j}^{2}-2 y_{i} y_{j}$, therefore $y_{i}^{2}+y_{j}^{2} \geq 2 y_{i} y_{j}$, with the equality holding if, and only if, $y_{i}=y_{j}$.

Therefore,

$$
\begin{gathered}
1=\left(\sum_{i=1}^{5} y_{i}\right) 2=\sum_{i=1}^{5} y_{i}^{2}+2 \sum_{\substack{i, j=1, i \neq j}}^{5} y_{i} y_{j} \leq \sum_{i=1}^{5} y_{i}^{2}+ \\
+2 \sum_{\substack{i, j=1, i \neq j}}^{5}\left(y_{i}^{2}+y_{j}^{2}\right) \\
=5 \sum_{i=1}^{5} y_{i}^{2} \text { or } \sum_{i=1}^{5} y_{i}^{2} \geq \frac{1}{5}
\end{gathered}
$$

with the equality holding if and only if $y_{1}=y_{2}=y_{3}=y_{4}=y_{5}=$ $\frac{1}{5}$. In the case of equality the first of formulas (5) gives that $X_{c}=7\left(\frac{1}{5}+\frac{2}{5}+\frac{3}{5}+\frac{4}{5}+\frac{5}{5}\right)-2=19$. Further, combining the inequality (6) with the second of formulas (5) one finds that $Y_{c} \geq \frac{3}{35}$ Therefore the unique minimum for $Y_{c}$ corresponds to the COG $F_{m}\left(19, \frac{3}{35}\right)$. The ideal case is when $y_{1}=y_{2}=y_{3}=y_{4}=0$ and $y_{5}=1$. Then from formulas (5) we get that $X_{c}=33$ and $Y_{c}=\frac{3}{7}$.Therefore the COG in this case is the point $F_{i}\left(33, \frac{3}{7}\right)$. On the other hand, the worst case is when $y_{1}=1$ and $y_{2}=y_{3}=y_{4}=y_{5}=0$. Then from formulas (5), we find that the COG is the point $F_{w}\left(5, \frac{3}{7}\right)$. Therefore the area where the COG $F_{c}$ lies is the area of the triangle $\mathrm{F}_{\mathrm{w}} \mathrm{F}_{\mathrm{m}} \mathrm{F}_{\mathrm{i}}$ (see Figure 3).
6. We formulate our criterion for comparing the performances of two (or more) different groups' as follows: From elementary geometric observations (see Figure 3) it follows that for two groups the group having the greater $X_{c}$ performs better. Further, if the two groups have the same $X_{c}$ $\geq 19$, then the group having the COG which is situated closer to $F i$ is the group with the greater $Y_{c}$. Also, if the two groups have the same $X_{c}<19$, then the group having the COG which is situated farther to $F w$ is the group with the smaller $Y_{c}$. Based on the above considerations it is logical to formulate our criterion for comparing the two groups' performance in the following form:

- Between two groups the group with the greater value of $X_{c}$ demonstrates the better performance.

- If two groups have the same $X_{c} \geq 19$, then the group with the greater value of $Y_{c}$ demonstrates the better performance.

- If two groups have the same $X_{c}<19$, then the group with the smaller value of $Y_{c}$ demonstrates the better performance.

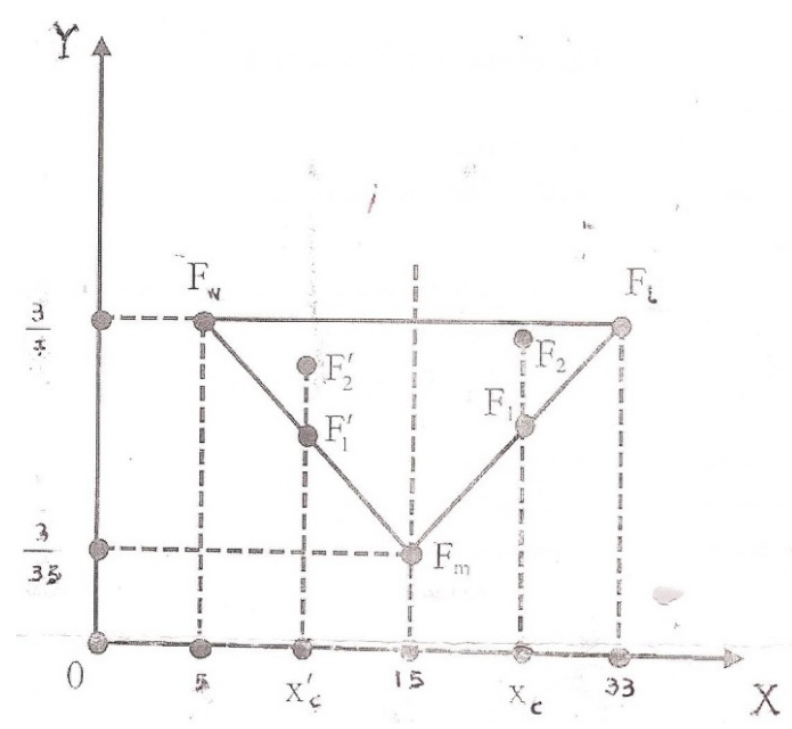

Figure 3. The area where the COG lies

REMARK: An alternative to the TRFAM approach is to consider isosceles triangles instead of trapezoids $[4,5]$. Then applying a similar argument as for the TRAFM above one finds that the coordinates of the COG of the resulting in this case scheme are calculated by the formulas

$$
X_{c}=\left(7 \sum_{i=1}^{5} i y_{i}\right)-2, Y_{c}=\frac{1}{5} \sum_{i=1}^{5} y_{i}^{2}
$$

(see [5]). An analogous criterion can be also obtained for comparing the performance of two (or more) different group of individuals'. We have called the above framework Triangular Fuzzy Assessment Model (TFAM).

\section{An Application}


The students of two different Departments of the School of Technological Applications (prospective engineers) of the Graduate T. E. I. of Western Greece achieved the following scores in the final common exam of the mathematics course of their first term of studies (the contents of the course were the same and the instructor was the same person for both Departments):

Department 1 ( $\left.\mathrm{D}_{1}\right)$ : 99(1 student), 83(2), 82(1), 74(10), 72(2), 70(1), 59(10), 55(2), 48(7), 45(2). Department $2\left(\mathrm{D}_{2}\right)$ : 85(2), 75(1), 62(2), 60(10), 52(1), 50(8), 25(4), 10(1).

The results of the students' performance are summarized in Table 1 below:

Table 1. The exam's results

\begin{tabular}{|c|c|c|}
\hline Grade & $\mathrm{D}_{1}$ & $\mathrm{D}_{2}$ \\
\hline $\mathrm{A}$ & 1 & 2 \\
\hline $\mathrm{B}$ & 3 & 1 \\
\hline $\mathrm{C}$ & 13 & 12 \\
\hline $\mathrm{D}$ & 12 & 9 \\
\hline $\mathrm{F}$ & 9 & 5 \\
\hline Total & 38 & 29 \\
\hline
\end{tabular}

The evaluation of the above results will be performed below using both traditional methods, based on principles of the classical (bivalent) logic, and fuzzy logic methods.

\subsection{Traditional Methods}

i) Calculation of the means: A straightforward calculation gives that the means of the students' scores are approximately 62.231 and 52.793 for $\mathrm{D}_{1}$ and $\mathrm{D}_{2}$ respectively. This shows that the mean performance was good (C) for the students of $\mathrm{D}_{1}$ and satisfactory (D) for the students of $\mathrm{D}_{2}$.

ii) Calculation of the GPA index: We recall that the Great Point Average (GPA) index is a weighted mean, where more importance is given to the higher scores by attaching greater coefficients (weights) to them (e.g. see [1]). In other words, the GPA index focuses on the quality performance of a student group.

Let us denote by $n_{A}, n_{B}, n_{C}, n_{D}$ and $n_{E}$ the numbers of students of a given group whose performance is characterized by A, B, C, D and F respectively and by $n$ the total number of students of the group. Then, the GPA index is calculated by the formula $\mathrm{GPA}=$ $\frac{0 n_{F}+n_{D}+2 n_{C}+3 n_{B}+4 n_{A}}{n}$. Obviously we have that 0 $\leq \mathrm{GPA} \leq 4$

In our case the above formula can be written as:

$$
\mathrm{GPA}=y_{2}+2 y_{3}+3 \mathrm{y}_{4}+4 y_{5}
$$

Then, using the data of Table 1 it is easy to check that the GPA for $\mathrm{D}_{1}$ is equal to $\frac{51}{38} \approx 1.342$ and for $\mathrm{D}_{2}$ is equal to $\frac{44}{29} \approx 1.517$. Therefore, since the values of the GPA index are less than the half of its maximal possible value, which is equal to 4 , the quality performance of both Departments was less than satisfactory. However, in contrast to their mean performances, the quality performance of $\mathrm{D}_{2}$ was better than the corresponding performance of $D_{1}$.

\subsection{Fuzzy Logic Methods}

In this paragraph we shall apply the fuzzy logic methods described in sections 2 and 3 of this paper as follows:

iii) The COG method: Observing the coefficients of the $y_{i}{ }^{\prime} s, \mathrm{i}=1,2,3,4,5$, in the first of formulas (3) and taking into account that, according to the criterion stated in section 2 , the COG's abscissa $x_{c}$ measures a student group's performance, it becomes evident that the COG method is also focused, as the GPA index does, on the student groups' quality performance.

In case of our classroom application taking into account the data of Table 1 and using the first of formulas (3) we find that $x_{c}=\frac{140}{76} \approx 1.842$ for $\mathrm{D}_{1}$ and $x_{c}=\frac{117}{58} \approx 2.017$ for $\mathrm{D}_{2}$. Since the above values of $x_{c}$ are less than the half of its value in the ideal case, which is equal to $\frac{9}{2}$ (see section 2), the quality performance of both Departments according was less than satisfactory. Further $\mathrm{D}_{2}$ demonstrated a better quality performance than $\mathrm{D}_{1}$.

iv) Application of TRFAM: Observing the coefficients of the $y_{i}$ 's, $\mathrm{i}=1,2,3,4,5$, in the first of formulas (5) it is easy to conclude that the TRFAM method is also focused on the student groups' quality performance.

In case of our classroom application taking into account the data of Table 1 and using the first of formulas (5) we find that $X_{c}=\frac{623}{39} \approx 15.974$ for $\mathrm{D}_{1}$ and $X_{c}=\frac{511}{29} \approx 17.621$ for $\mathrm{D}_{2}$. In this case the value of $X_{c}$ for $\mathrm{D}_{1}$ is less than the half of its value in the ideal case, which is equal to 33 (see section 3 ). This shows that the quality performance of $D_{1}$ according was less than satisfactory. On the contrary, since the value of $X_{c}$ for $\mathrm{D}_{2}$ is greater than the half of its value in the ideal case, the quality performance of $\mathrm{D}_{2}$ was more than satisfactory.

REMARK: Analogous results are obtained if, instead of formulas (5), we apply formulas (7) of TFAM.

\subsection{Comparison of the Used Assessment Methods}

In paragraphs 4.1 and 4.2 we have applied four in total assessment methods. The first of these methods measures the mean performance of a group, while the other three methods (GPA, COG and TRFAM) measure its quality performance by assigning greater coefficients (weights) to the higher scores. The coefficients attached to the $y_{i}$ 's in these three methods -see formula (8) and the first of formulas (3) and (5) respectively- are presented in the following Table 2.

From Table 2 it becomes evident that TRFAM assigns 
greater coefficients to the higher with respect to the lower scores than COG and also COG does the same thing with respect to GPA. In other words TRFAM is more accurate than COG, and COG is more accurate than GPA for measuring the quality performance of a student group.

Table 2. Weight coefficients of the yi's

\begin{tabular}{|c|c|c|c|}
\hline $\mathrm{y}_{\mathrm{i}}$ & GPA & COG $\left(\mathrm{x}_{\mathrm{c}}\right)$ & TRAFM $\left(\mathrm{X}_{\mathrm{c}}\right)$ \\
\hline $\mathrm{y}_{1}$ & 0 & $1 / 2$ & 7 \\
\hline $\mathrm{y}_{2}$ & 1 & $3 / 2$ & 14 \\
\hline $\mathrm{y}_{3}$ & 2 & $5 / 2$ & 21 \\
\hline $\mathrm{y}_{4}$ & 3 & $7 / 2$ & 28 \\
\hline $\mathrm{y}_{5}$ & 4 & $9 / 2$ & 35 \\
\hline
\end{tabular}

This explains why in our classroom application the quality performances of $D_{1}$ and $D_{2}$ were found to be less than satisfactory by applying the GPA and COG methods, while the application of the TRAFM method has demonstrated a more than satisfactory quality performance for $\mathrm{D}_{2}$ and less than satisfactory for $\mathrm{D}_{1}$.

One should also mention that, while $\mathrm{D}_{2}$ demonstrated in all cases (GPA, GOG and TRAFM) a better quality performance than $\mathrm{D}_{1}$, in contrast to the mean performance of $\mathrm{D}_{1}$, which was found to be better than the corresponding performance of $\mathrm{D}_{2}$ (first method of paragraph 4.1).

In concluding, it is suggested to the user of the above four assessment methods to choose the one that fits better to his/her personal goals.

\section{Conclusions and Discussion}

The methods for assessing a group's performance (in any human activities) usually applied in practice are based on the principles of the bivalent logic (yes-no). However, fuzzy logic, due to its nature of characterizing a situation with multiple values by using linguistic variables, offers a wider and richer field of resources for this purpose. This gave us the impulsion to introduce principles of fuzzy logic for developing the TRFAM approach for the individuals' assessment. The TRFAM is actually a more sensitive version of the COG fitting better to the ambiguous cases of students' scores lying at the limits between two different grades. We also presented some applications in which we have compared the outcomes of TRFAM approach with the corresponding outcomes of the COG technique and of other traditional assessment methods (the calculation of the means and the GPA index).

However, there is a need for more classroom applications to be performed in the future for obtaining safer statistical data. On the other hand, since the TRFAM approach appears to have the potential of a general assessment method, our future research plans include also the effort to apply this approach for assessing the individuals' performance in several other human activities.

\section{REFERENCES}

[1] Grade Point Average Assessment, available in the Web at: $\mathrm{http} / /$ www.swinburne.edu.au/student-administration/assess ment/gpa.html, visited October 15, 2014.

[2] Klir, G. J. \& Folger, T. A., Fuzzy Sets, Uncertainty and Information, Prentice-Hall, London, 1988.

[3] Subbotin, I. Ya. Badkoobehi, H., Bilotckii, N. N., Application of fuzzy logic to learning assessment, Didactics of Mathematics: Problems and Investigations, 22, 38-41, 2004.

[4] Subbotin, I. Ya., Bilotskii, N. N., Triangular fuzzy logic model for learning assessment, Didactics of Mathematics: Problems and Investigations, 41, 84-88, 2014.

[5] Subbotin, I. Ya. , Voskoglou, M. Gr., A Triangular Fuzzy Model for Assessing Students' Critical Thinking Skills, International Journal of Applications of Fuzzy Sets and Artificial Intelligence, 4, 173-186, 2014.

[6] Subbotin, I. Ya., Trapezoidal Fuzzy Logic Model for Learning Assessment, arXiv 1407.0823[math.GM], submitted on July 1, 2014

[7] Subbotin, I. Ya, Voskoglou, M. Gr., Fuzzy Models for Learning Assessment, arXiv: 1410.4497 [math.OC], submitted on October 15, 2014.

[8] van Broekhoven, E., De Baets, B., Fast and accurate centre of gravity defuzzification of fuzzy system outputs defined on trapezoidal fuzzy partitions, Fuzzy Sets and Systems, 157(7), 904-918, 2006.

[9] Voskoglou, M. Gr., Subbotin, I. Ya., Fuzzy Models for Analogical Reasoning, International Journal of Applications of Fuzzy Sets and Artificial Intelligence, 2, 19-38, 2012.

[10] Voskoglou, M. Gr , Fuzzy measures for students' mathematical modelling skills, International Journal of Fuzzy Logic Systems, 2(2), 13-26, 2012.

[11] Voskoglou, M. Gr , Subbotin, I. Ya, Dealing with the Fuzziness of Human Reasoning, International Journal of Applications of Fuzzy Sets and Artificial Intelligence, 3, 91-106, 2013.

[12] Voskoglou, M. Gr., Assessing the Players' Performance in the Game of Bridge: A fuzzy Logic Approach, American Journal of Applied Mathematics and Statistics, 2(3), 115-120, 2014.

[13] Wikipedia, Center of mass: Definition, available in the Web at: http://en.wikipedia.org/wiki/Center_of_mass\#Definition , visited on October 10, 2014.

[14] Wikipedia, Trapezoid: Other properties, available in the Web at: http://en.wikipedia.org/wiki/trapezoid\#other_properties , visited on October 10, 2014.

[15] Wikipedia, Center of mass: A system of particles, available in the Web at:

http://en.wikipedia.org/wiki/Center_of_mass\#A_system_of_ particles, visited on October 10, 2014. 